\title{
EL TRABAJO COLABORATIVO EN PRIMARIA Y SECUNDARIA: \\ DIFERENCIAS SEGÚN SEXO Y CURSO
}

\section{Collaborative work in primary and secondary education: differences according to gender and academic course}

\author{
Trabalho colaborativo na primária e secundária: \\ diferenças de acordo com o sexo eo curso \\ Amaya Epelde Larrañaga ${ }^{1}$ \\ Ramón Chacón Cuberos ${ }^{2}$ \\ José Antonio Oñederra Ramírez ${ }^{3}$
}

Recibido: $1^{\circ}$ de octubre de 2018. Corregido: 13 de junio de 2019. Aprobado: 30 de junio de 2019.

\section{Resumen}

El objetivo principal de la educación primaria y secundaria consiste en facilitar a los jóvenes una formación plena que les permita desempeñar una futura vida personal y profesional de calidad. Durante la vida profesional de una persona, el trabajo en equipo es una herramienta muy utilizada debido a las grandes ventajas que proporciona a nivel individual, grupal, social y profesional, pero ello exige una preparación previa

1 Doctora Europea por la Universidad de Granada. Profesora del Departamento de Didáctica de la Expresión Musical, Plástica y Corporal en el Campus Universitario de Melilla. Líneas de investigación: Creatividad musical en primaria, la interculturalidad en los centros educativos y la Creatividad Musical para la interculturalidad de los alumnos. Correo electrónico: aepelde@ugr.es

2 Doctor en Ciencias de la Educación por la Universidad de Granada. Profesor en el Departamento de Didácticas Integradas de la Facultad de Ciencias de la Educación de la Universidad de Huelva. Líneas de investigación: educación física, salud, deporte, actividad física. Correo electrónico: ramon.chacon@ddi.uhu.es

${ }^{3}$ Doctor en Psicología por la Universidad del País Vasco. Profesor en el Instituto de Educación Secundaria rỉidebietaò de San Sebastián. Líneas de investigación: violencia entre iguales, bullying y cyberbullying. Correo electrónico: joxeano@hotmail.com

Acta Sociológica núm. 79, maYo-agosto de 2019, pp. 11-32. 
que se debe adquirir durante la etapa escolar. En este manuscrito presentamos el estudio que hemos realizado sobre el trabajo en equipo que se lleva a cabo en dos centros de Educación Primaria y Secundaria de la Ciudad Autónoma de Melilla (España). Se pretenden ver las diferencias existentes en este aspecto según sexo, curso y tipo de centro. Los resultados obtenidos demuestran que las mujeres son más responsables que los hombres al llevar a cabo un trabajo en grupo y que a mayor edad o curso superior, disminuye la motivación e implicación en el trabajo colaborativo.

Palabras clave: Trabajo en Equipo, Trabajo Social, Aprendizaje Colaborativo, Educación Primaria, Educación Secundaria, Prevalencia.

\begin{abstract}
The aim of the Primary and Secondary Education consists of facilitating to adolescents a full training that allows them to carry out a qualified personal and professional future life. At that time, the teamwork is a very used tool due to the big advantages that it provides to individual, grupal, social and professional levels, but it is required a previous preparation that it is necessary to acquire during the school stage. In this research, we analize the Teamwork in two centers of Primary and Secondary Education of the Autonomous City of Melilla (Spain). We need to see the differences according to sex, course and type of center. The results demonstrate that in a teamwork the women are more responsible than the men. Also, with the increase in the age, the motivation and implication in the Collaborative Work diminishes.
\end{abstract}

Key words: Teamwork, Social Work, Collaborative Learning, Primary Education, Secondary Education, Prevalence.

\title{
Resumo
}

O objetivo principal da Educação Primária e Secundária é proporcionar aos jovens uma educação completa que lhes permita ter uma vida futura pessoal e profissional de qualidade. Durante a vida profissional de uma pessoa, o trabalho em equipe é uma ferramenta muito utilizada devido às grandes vantagens que proporciona ao nível individual, grupal, social e profissional, mas isso requer um preparo prévio que deve ser adquirido durante a etapa escolar. Neste manuscrito apresentamos o estudo que fizemos sobre o trabalho em equipe, que ocorre em dois centros de Educação Primária e Secundária da cidade autônoma de Melilla (Espanha). Pretendemos ver as diferenças neste aspecto de acordo com sexo, curso e tipo de centro. Os resultados obtidos mostram que as mulheres são mais responsáveis do que os homens na realização de um trabalho em equipe e que em uma idade maior ou curso superior, a motivação e o envolvimento no Trabalho Colaborativo diminui.

Palavras chave: trabalho em equipe, trabalho social, aprendizagem colaborativo, Educação Primária, Educação Secundaria, prevalencia. 


\section{Resumen de metodología, desarrollo y conclusiones de la investigación}

El trabajo colaborativo es una estrategia metodológica que se utiliza en todas las etapas educativas con el fin de conseguir un aprendizaje significativo de las materias. Pero además de esta finalidad, tiene otra aún más marcada como es el aspecto social, es decir, las habilidades y capacidades sociales que desarrolla. El aprendizaje colaborativo que se lleva a cabo en los centros educativos a través del trabajo en equipo potencia las cualidades sociales y culturales de la persona y la prepara para un futuro prometedor y exitoso. Por esta razón, en este manuscrito analizamos el trabajo colaborativo en dos centros de educación primaria y secundaria ubicados en la Ciudad Autónoma de Melilla (España). Pretendemos observar las diferencias existentes según género, curso y tipo de centro a la hora de utilizar esta herramienta. Para ello, ponemos en marcha un cuestionario de elaboración propia compuesto de 14 items, que fue validado por expertos en la materia.

La muestra estuvo compuesta por 227 adolescentes y el análisis estadístico se realizó mediante el software IBM SPSS®22.0. Los descriptivos básicos fueron analizados mediante frecuencias y medias, mientras que para las relaciones entre variables se emplearon tablas de contingencia. La fiabilidad fue valorada mediante el coeficiente alfa de Cronbach.

Los resultados obtenidos revelan que las féminas están más motivadas y son más responsables que los varones a la hora de llevar a cabo un trabajo en grupo y que cuanto más elevado sea el curso escolar, es menor la motivación hacia esta herramienta metodológica.

\section{Fundamentos}

La sociedad actual se caracteriza por estar situada en un ambiente altamente competitivo y complejo en el que se necesitan herramientas factibles para lograr resultados óptimos. Una de las herramientas que permiten el éxito es el trabajo colaborativo. En nuestra vida diaria encontramos, con frecuencia, la necesidad de realizar tareas de distinta índole que requieren de una estrecha colaboración entre varios participantes 
para que su resultado sea óptimo y rápido. Igualmente, en cualquier empresa o institución, se trabaja de manera muy frecuente en equipo y se consiguen objetivos en un corto tiempo que de manera individual serían improbables e incluso imposibles de lograr. Tal como dice Estefanía Dávila Martín, ${ }^{4}$ vivimos en una era en la que todo el mundo trata de r̃anar tiempo, de medirlo y calcularlo con precisión para acercar el futuro a una mayor velocidadò Así, obtenemos resultados mejores cualitativamente y mayores de manera cuantitativa en el menor tiempo posible.

Para llevar a cabo este tipo de colaboración entre compañeros, es preciso adquirir previamente una formación que facilite su desempeño. Tal como dice Moreno Peláez, ${ }^{5}$ el aprendizaje organizativo es la herramienta fundamental para conseguir ventajas competitivas e insta a que a través de la inteligencia que se adquiere por medio de dicho aprendizaje, se pueda crear un espacio que determine el comportamiento hacia la cooperación, hacia la percepción del bien común. De ahí que la autora otorgue tanta importancia a la acción social que se lleve a cabo para conseguir el éxito individual y grupal en cualquier campo.

Este aprendizaje debe ser adquirido previo a su práctica y cuanto más temprana sea la edad en la que se alcance, más y mejor quedará interiorizado. La educación primaria y secundaria son dos etapas educativas muy importantes en las que, de manera globalizada, se prepara al alumnado para su vida adulta profesional y personal. El aprendizaje colaborativo es una de las ramas de esa formación y por esta razón es muy importante y necesario que los alumnos trabajen en grupo en sus actividades escolares.

Es obligado referirse a las investigaciones de Piaget $^{6}$ en torno al papel de la actividad y de la interacción social en el desarrollo intelectual; dichas investigaciones le convirtieron en un decidido defensor del trabajo por equipos. En fechas posteriores, existe un claro consenso entre investigadores y pedagogos en cuanto a la importancia de las estrategias

${ }^{4}$ Dávila Martín, Estefanía (2016), r̃Rápido a ninguna parte. Consideraciones en torno a la aceleración del tiempo socialò Acta Sociológica, núm. 69, CES, FCPyS UNAM, p. 61.

${ }^{5}$ Moreno Peláez, Florentina (1997), ñAprendizaje organizativo y generación de competenciasò Revista Española de Investigaciones Sociológicas, núm. 77, CIS, Madrid, pp. 247-261.

${ }^{6}$ Piaget, Jean (1969), Psicología y pedagogía, Ariel, Barcelona. 
colaborativas en el aula para contribuir a crear un buen clima de aula y a mejorar el aprendizaje en cualquier tipo de tarea o contenido de enseñanza y en cualquier nivel académico. El convivir con personas diferentes y la necesidad de colaboración para sobrevivir o realizar tareas en común obliga a potenciar habilidades y capacidades específicas que han de desarrollar los estudiantes en una etapa temprana de su vida. León, Gozalo y Polo, ${ }^{7}$ y Chacón-Cuberos, Castro-Sánchez, González-Campos y Zurita-Ortega ${ }^{8}$ aseguran que es necesario recurrir a las técnicas y programas de aprendizaje cooperativo que existen en los centros educativos para disminuir agresiones entre menores, sobre todo la exclusión social que, por desgracia, es una de las modalidades de agresión más frecuentes en los centros educativos. Estos programas permiten mejores resultados de aprendizaje unidos a un incremento cualitativo de las relaciones sociales entre los estudiantes, lo que indudablemente repercutirá en la adopción del modus operandi correcto en su vida laboral y profesional futura obteniendo resultados óptimos.

Para terminar esta introducción queremos aclarar que en nuestro trabajo no hacemos diferencias entre los términos trabajo en grupo, trabajo en equipo y trabajo colaborativo, teniendo en cuenta que en la literatura consultada existen diferentes criterios al respecto. Algunos autores utilizan como sinónimos dichos términos y otros exponen diferencias entre ellos. ${ }^{9}$ Recientemente, los autores Rosenfield, Newell, Zwolski y Benishek ${ }^{10}$ y Pang, Lau, Seah, Cheong y Low ${ }^{11}$ los han usado indistintamente en sus investigaciones, y siguiendo esta línea, no entraremos a debatir las diferencias y similitudes entre ellos y los trataremos como términos similares.

${ }^{7}$ León, Benito, Margarita Gozalo, María Isabel Polo (2012), r̃Aprendizaje cooperativo y acoso entre igualesò, Infancia y aprendizaje, vol. 35, núm. 1, Fundación Infancia y Aprendizaje, España, pp. 23-35.

${ }^{8}$ Chacón-Cuberos, Ramón, Manuel Castro-Sánchez, Gloria González-Campos, Félix Zurita-Ortega (2018), ñ ictimización en la escuela, ocio digital e irritabilidad: análisis mediante ecuaciones estructuralesò RELIEVE, vol. 24, núm. 1, MIDE, Valencia. doi: 10.7203/relieve.24.1.12614.

${ }_{9}$ Gómez Múgica, Aleida, Heriberto Acosta Rodríguez (2003), ñAcerca del trabajo en grupos o equiposò $A C I M E D$, vol. 11, núm. 6, Infomed, Cuba.

${ }^{10}$ Rosenfield, Sylvia, Markeda Newell, Scott Zwolski, Lauren E. Benishek (2018), íEvaluating problem-solving teams in Kï 12 schools: do they work? ò American Psychologist, v. 73, n. 4, American Psychological Association, Washington D.C., pp. 407-419. 


\section{Ventajas del trabajo colaborativo}

Desde hace muchos años, en el ámbito de la educación se defiende el uso de metodologías nuevas con la finalidad de lograr un aprendizaje creativo y constructivista, que despierte un espíritu crítico y emprendedor y desarrolle la creatividad entre los alumnos. Actualmente, se echa todavía de menos este tipo de metodología abierta, dialogante, experimental y con iniciativa que permita al alumno expresar con libertad sus opiniones y experiencias. El trabajo educativo realizado en grupo requiere diálogo entre los miembros del equipo y compartir ideas entre compañeros, escuchándolos y respetándolos; permite construir aprendizajes nuevos, transformar pensamientos, movilizarse para conseguir nuevas metas, mejorar niveles de inteligencia, desarrollar capacidades y habilidades sociales, y mejorar las relaciones personales entre los miembros del grupo.

Andreu-Andrés y García-Casas, ${ }^{12}$ aseguran que ña pasividad, el individualismo, la competitividad y la memorización se sustituyen por cooperación y responsabilidad para llevar a cabo la tarea asignadaò

Según Goikoetxea y Pascual, ${ }^{13}$ se puede considerar el aprendizaje colaborativo como un modelo en el que un grupo de estudiantes, más o menos reducido, con diferentes niveles y habilidades, comparte una tarea específica y todos se responsabilizan no sólo de su propio aprendizaje, sino también del de sus compañeros; de este modo se desarrolla el pensamiento crítico de los participantes, estrechan entre ellos relaciones sociales, conforman la búsqueda de fines comunes y comparten su consecución. Después del trabajo realizado, el equipo evalúa sus propias acciones y resultados para aprender de sus errores.

${ }^{11}$ Pang, Christopher, Jesslyn Lau, Chong Poh Seah, Linda Cheong, Audrey Low (2018), ñSocially challenged collaborative learning of secondary school students in Singaporeò Education Sciences, v. 8, n. 24, Switzerland.

12 Andreu-Andrés, María Ángeles, Miguel García-Casas (2008), ña autoevaluación del trabajo en grupo en la clase de lengua extranjera: investigación de campoò $L a$ evaluación en el aprendizaje y la enseñanza del español como LE/L2, Susa Pastor y Santiago Roca Editores, Universidad de Alicante, Alicante, p. 146.

${ }^{13}$ Goikoetxea, Edurne, Gema Pascual (2002), ñAprendizaje cooperativo: Bases teóricas y hallazgos empíricos que explican su eficaciaò Educación $X X I$, núm. 5, UNED, España, pp. 227-247. 
Con todo lo dicho, vemos que el aprendizaje colaborativo aporta a la persona una formación íntegra tanto a nivel social como cultural. Por esta razón, Bernal, Epelde, Gallardo y Rodríguez ${ }^{14}$ defienden el trabajo conjunto y colaborativo tanto entre los alumnos como entre los docentes. Aseguran que un trabajo en equipo bien realizado mejora la calidad en todas las ramas y facilita el aprendizaje.

En esta línea, García-Valcárcel, Basilotta y López ${ }^{15}$ defienden las TIC como recurso para facilitar el trabajo colaborativo porque dicen que permite construir entornos de aprendizaje social donde el diálogo y el aprendizaje se ponen en marcha de manera conjunta. Estas autoras aseguran que el trabajo colaborativo permite desarrollar las habilidades sociales, la resolución de problemas, la autonomía, la responsabilidad, la capacidad de reflexión y la iniciativa.

Sin embargo, es necesario tener en cuenta las limitaciones que presenta el trabajo colaborativo en los centros educativos; son aspectos docentes y pedagógicos que requieren de nuestra atención. García-Valcárcel, Basilotta y López ${ }^{16}$ revelan que a pesar de los años transcurridos y de la infraestructura incorporada, los maestros emplean más tiempo en preparar y llevar a cabo las actividades grupales que las individuales, y que durante éstas, existe más descontrol en la clase, la organización se resiente, los miembros de los grupos se implican unos más que otros y a todo lo anterior se añade la dificultad del profesorado para evaluar. Esto trae como consecuencia una disminución del temario impartido, ya que el proceso es más lento.

Pensamos que estos problemas se pueden resolver a través de cursos de formación para docentes sobre trabajos colaborativos, su importancia y sus ventajas, y a pesar de estos inconvenientes, estamos seguros de que

${ }^{14}$ Bernal Vázquez, Julia, Amaya Epelde Larrañaga, Miguel Ángel Gallardo Vigil, África Rodríguez Blanco (2012), r̃La enseñanza colaborativa en Educación Primaria. Un estudio sobre el trabajo conjunto de los docentes de música e inglés para mejorar la formación del alumnadoò DEDiCA. Revista de Educação e Humanidades, núm. 2, Portugal, pp. 295-306.

${ }^{15}$ García-Valcárcel, Ana, Verónica Basilotta, Camino López (2014), raas TIC en el aprendizaje colaborativo en el aula de Primaria y Secundariaò Comunicar, vol. 42, núm. 21, España, pp. 65.

${ }^{16}$ Idem. 
siempre serán mayores y más numerosos sus beneficios; por tanto, creemos que es imprescindible la formación en esta rama y su práctica tanto en la etapa escolar como en la profesional.

\section{Material y método}

\section{Objetivos}

En este trabajo se analiza el trabajo en equipo que se lleva a cabo en dos centros de primaria y secundaria, uno privado y otro concertado ubicados en la Ciudad Autónoma de Melilla (España). Se ven las diferencias existentes según sexo, curso y tipo de centro.

La finalidad principal consiste en comprobar si se está teniendo éxito en la enseñanza-aprendizaje del trabajo en grupo y de las normas de aplicación del mismo, entre los alumnos de edades comprendidas entre los 11 y los 14 años.

Los centros que han participado son el Instituto Público de Educación Secundaria Enrique Nieto en el que se ha trabajado con varias líneas del curso de $1^{\circ}$ de ESO, y el Colegio Concertado La Salle ŕEl Carmenòen el que se ha trabajado en las dos líneas existentes en cada uno de los cursos de $6^{\circ}$ de Primaria y $1^{\circ}$ de ESO. En ambos centros la multiculturalidad y la diversidad cultural resulta palpable, donde la población musulmana y la cristiana se encuentran en un porcentaje igualado.

\section{Diseño y participantes}

Se llevó a cabo un estudio no experimental, ex post-facto, de carácter descriptivo y medición única en un único grupo. La muestra estuvo compuesta por 227 adolescentes de la ciudad autónoma de Melilla, con una representación por sexo del $45.4 \%(n=103)$ de varones y del $54.6 \%$ $(n=124)$ de mujeres y una edad comprendida entre los 11 y 15 años (12.06 \pm 0.77 ). Para el curso 2017/2018, 2536 alumnos estaban matriculados en Melilla en sexto curso de Educación Primaria $(n=1165)$ y primer curso de ESO $(n=1371)$. Se estableció una muestra representativa de 227 escolares (error muestral de $0.05 ; \mathrm{IC}=90 \%$ ). La distribución de los centros participantes fue la siguiente: IES Enrique Nieto con un 46,3\% $(n=105)$ de alumnado y La Salle con el $53.7 \%(n=122)$. Para la selección de los 
participantes, los cuales tomaron parte de forma voluntaria, se consideró una aleatorización por grupos naturales siguiendo los criterios de MerinoMarban, Mayorga-Vega, Fernández-Rodríguez, Estrada y Viciana. ${ }^{17}$

\section{Instrumentos}

Test de trabajo en equipo. Se utiliza un cuestionario de elaboración propia, el cual fue validado mediante panel de expertos. Este cuestionario se compone de 14 ítems que puntúan diferentes aspectos de la capacidad de los encuestados para trabajar en grupo (p. e. r̃Contribuyes a que la comunicación entre los miembros del grupo sea clara y fluida). Estos son puntuados mediante una escala tipo Likert de cuatro opciones donde $1=$ Nunca y $4=$ Siempre. Para este instrumento se obtuvo una consistencia interna de á $=0.806$, la cual es aceptable.

Hoja de auto-registro. Se emplea un cuestionario tipo ad hoc para el registro de variables de tipo sociodemográfico, tales como el sexo, la edad, el centro educativo o el curso.

\section{Procedimiento}

En primer lugar, se procedió a solicitar la colaboración de los centros educativos a través de la presencia directa en ellos de uno de los autores de este manuscrito. En una reunión mantenida con el equipo directivo de los centros que participaron en el estudio, se les detalló la naturaleza y objetivos del estudio a realizar. Posteriormente, se solicitó el consentimiento informado de los responsables legales de los adolescentes a través de un modelo de autorización.

La aplicación de los instrumentos se realizó en horario lectivo en los centros detallados sin que ocurriese ningún tipo de incidencia. Asimismo, los investigadores estuvieron presentes durante el proceso con el fin de asegurar una correcta aplicación de los mismos. Destacar que se aseguró el anonimato a todos los estudiantes, los cuales participaron de manera

17 Merino-Marban, Rafael, Daniel Mayorga-Vega, Emilio Fernández-Rodríguez, Francisco Vera Estrada, Jesús Viciana (2015), r̃Effect of a physical education-based stretching programme on sit-andreach score and its posterior reduction in elementary schoolchildrenò European Physical Education Review, n. 21, UK, pp. 83-92. 
voluntaria y respetando el acuerdo sobre ética de investigación de Helsinki en 1975.

\section{Análisis de datos}

El análisis estadístico se realizó mediante el software IBM SPSS®22.0. Los descriptivos básicos fueron analizados mediante frecuencias y medias, mientras que para las relaciones entre variables se empleó tablas de contingencia. La fiabilidad interna de los instrumentos empleados fue valorada mediante el coeficiente alfa de Cronbach, fijando el Índice de confiabilidad en el $95.5 \%$. La fiabilidad fue establecida en $p \leq 0.05$.

\section{Resultados}

El trabajo en grupo según el sexo de la muestra estudiada (Tabla I en Anexo 1) reveló diferencias estadísticamente significativas en cinco categorías y se detectó una tendencia clara en otra de ellas. De forma global, se observan puntuaciones medias más elevadas en cuanto a mejores aptitudes en el trabajo en grupo en las chicas participantes con respecto a los chicos. Véase el ítem 2: ñener claras las responsabilidades como miembro del equipoò un $8.7 \%$ de los varones aseguran no tener nunca claras sus responsabilidades, frente a un $0 \%$ de chicas quienes según nuestros datos, son más responsables a la hora de trabajar en grupo; en el ítem 3 , r̃contribuir a que exista un clima de trabajo agradableòson también un $6.8 \%$ de los chicos quienes no contribuyen nunca a alcanzar este logro, frente a un $0.8 \%$ de chicas; en el ítem 6 , r̃gestionar los conflictos de manera abiertaòson otra vez un $39.5 \%$ de las mujeres quienes lo hacen siempre, frente a un $25.2 \%$ de los varones. En este ítem, un 15.5\% de los hombres dicen no gestionar nunca los conflictos de manera abierta, frente a un $6.5 \%$ de mujeres que tampoco lo hace nunca. También son más las mujeres que los hombres $(96.8 \%$ vs $91.3 \%$ ), quienes ayudan a generar alternativas de solución ante los problemas (item 8$)$. También son ellas $(96.8 \%$ vs $88.4 \%)$, las que trabajan mejor analizando en grupo las posibles consecuencias de cada alternativa y ocurre lo mismo con su participación en el desarrollo en grupo de planes alternativos de trabajo (96.8\% vs $91.3 \%$ ). Según nuestros datos, las mujeres son más responsables que los hombres en el trabajo en grupo. 
En la relación entre el trabajo en grupo y el tipo de colegio, no se obtuvieron diferencias estadísticamente significativas en las distintas variables del trabajo en equipo de los alumnos respecto a si el centro es público o privado concertado. Pero cabe señalar que los alumnos del centro público reflejaron valores más elevados en trabajo grupal que los del colegio privado concertado.

La relación entre trabajo en grupo y curso se refleja en la tabla ॥ (ANEXO II), mostrándose una diferencia estadísticamente significativa con una puntuación superior en 6을 de primaria respecto a 1ํ de Eso en la categoría de gestionar los conflictos pensando más en las ideas que en las personas (80\% vs $72.5 \%$ ). Un $41.7 \%$ de los alumnos de 60 de primaria frente a un $18.6 \%$ de $1^{\circ}$ de ESO, aseguran gestionar los conflictos pensando más en las ideas que en las personas en la franja de valor r̃siempreò Sin embargo, en la pregunta 4 , sobre escuchar a los demás cuando se trabaja en grupo, encontramos una pequeña tendencia a que en $1^{\circ}$ de Eso se escucha más $(98.1 \%$ vs $95 \%)$. Es una tendencia muy ambivalente ya que en la franja de valor î́iempreòse observa que son los de 60 de primaria los que escuchan más $(73.3 \%$ vs $59 \%)$. Sin embargo, en la franja de riBastantes vecesòocurre lo contrario, son los de $1^{\circ}$ de Eso los más oyentes (28.3\% vs $15 \%$ ). En la categoría 10 îParticipas en el desarrollo en grupo de planes alternativos de trabajoòson los de $6^{\circ}$ de primaria (58.3\%) los más participativos en la franja de valor r̃siempreò En este sentido, se revela que en general los participantes de 6 o de primaria tienen mejores aptitudes para trabajar en grupo que los de $1^{\circ}$ de ESO, aunque las diferencias no son muy significativas.

\section{Discusión y conclusiones}

El presente estudio persigue analizar en una muestra de adolescentes de 6 을 curso de primaria y $1^{\circ}$ de la ESO, las relaciones existentes entre las variables de interés como es el sexo, la edad y la tipología del centro educativo en el que se encuentran escolarizados con el trabajo en grupo desempeñado en el mismo, para comprobar si los alumnos están adoptando las habilidades y capacidades intelectuales y sociales necesarias para llevar a cabo un buen trabajo en equipo.

Al realizar el presente estudio se ha constatado que las chicas 
estudiantes presentan en general aptitudes de mayor responsabilidad, sobre todo en las variables de tener claras las funciones del equipo, contribuir a que exista un clima de trabajo agradable, gestionar los conflictos de manera abierta, ayudar a generar alternativas de solución ante los problemas, analizar en grupo las posibles consecuencias de cada alternativa y participar en el desarrollo en grupo de planes alternativos de trabajo. En este sentido, este estudio presenta una línea similar a la de otros trabajos realizados en el contexto nacional e internacional en la última década. El estudio de Barbería ${ }^{18}$ también revela que las chicas se presentan más receptivas al trabajo en grupo que los chicos. El último informe $\mathrm{PISA}^{19}$ constata que las chicas son más colaborativas que los chicos en el grupo de aula, obtienen mejor puntuación en trabajo en equipo. En todos los países de la Organización para la Cooperación y Desarrollo Económico (OCDE), las chicas tienen más habilidades para colaborar que los chicos. Pang, Lau, Seah, Cheong y Low ${ }^{20}$ manifiestan que existen diferencias de género en las aptitudes de trabajo en equipo. En la misma línea, Froiland y $\operatorname{Oros}^{21}$ y Polo, Mendo, Fajardo y León, ${ }^{22}$ aseguran que las chicas presentan mayores niveles de motivaciones autodeterminadas e intrínsecas en comparación con los chicos. Todo lo dicho significa que las féminas se implican más que los varones en el aprendizaje cooperativo. ${ }^{23}$ En cambio, los chicos

18 Barbería, Garbiñe (2012), ñTrabajo colaborativoò Trabajo Fin de Master en la Universidad Pública de Navarra, Master Universitario en Formación del Profesorado en Educación Secundaria, en: https://academicae.unavarra.es/bitstream/handle/2454/15005/ 58502 Barberia\%20P\%C3\%A9rez\%2 C\%20Garbi\%C3\%B 1e.pdf? sequen ce $=1$ \&isAllowed $=y$

${ }^{19}$ PISA (2015), en: http://www.mecd.gob.es/dctm/inee/internacional/pisa2015/ pisa2015preliminarok.pdf?documentld $=0901 \mathrm{e} 72 \mathrm{~b} 8228 \mathrm{~b} 93 \mathrm{c}$

${ }^{20}$ Pang, Christopher, Jesslyn Lau, Chong Poh Seah, Linda Cheong, Audrey Low (2018), ñ Socially challenged collaborative learning of secondary school students in Singaporeò Education Sciences, v. 8, n. 24, Switzerland.

${ }^{21}$ Froiland, John Mark, Emily Oros (2014), ñntrinsic motivation, perceived competence and classroom engagement as longitudinal predictors of adolescent reading achievementò Educational Psychology, v. 34, n. 2, UK, pp. 119-132.

22 Polo, María Isabel, Santiago Mendo, Fernando Fajardo, Benito León (2017), Ĩna intervención en aprendizaje cooperativo sobre el perfil del observador en la dinámica bullyingò Universitas Psychologica, vol. 16, núm. 1, Colombia, pp. 1-13.

${ }^{23}$ Kibirige, Israel, Moyahabo Jeridah Lehong (2016), ñThe effect of cooperative learning on grade 12 learnersôperformance in projectile motions, South Africaò Eurasia Journal of Mathematics, Science \& Technology Education, v. 12, n. 9, UK, pp. 2543-2556. 
suelen estar extrínsecamente motivados de tal forma que otorgan un mayor valor a la competencia y, por tanto, se responsabilizan menos en el trabajo colaborativo. Siguiendo la misma línea de resultados, Garaigordobil y Maganto ${ }^{24}$ manifiestan que las chicas tienen mayores puntuaciones en estrategias cooperativas-positivas; mientras que los chicos, en las agresivas.

En esta línea, Choi, Johnson y Johnson ${ }^{25}$ y Zych, Beltrán-Catalán y Ortega-Ruiz ${ }^{26}$ demuestran que la intención de agredir es opuesta a la predisposición a cooperar, que los agresores tienen escaso comportamiento prosocial porque sus capacidades y emociones sociales y su conciencia social son muy escasas, y según Zischka ${ }^{27}$ cuanto más amplio y cercano sea el clima ambiental en el que se mueva una persona, ésta desarrollará en mayor medida su comportamiento prosocial. Todo ello nos hace pensar que si los chicos tienen menos aptitudes que las chicas para la cooperación, que es lo que obtenemos en las investigaciones nombradas y en la nuestra propia, va a ser necesario trabajar esta faceta especialmente con los varones. Tengamos en cuenta que en el estudio realizado por Van Ryzin y Roseth, ${ }^{28}$ el trabajo cooperativo reduce la victimización, mejora las relaciones sociales, desarrolla la empatía, y la inteligencia emocional, y León, Polo, Gozalo y Mendo ${ }^{29}$ aseguran que el aprendizaje colaborativo puede ser eficaz contra la exclusión social.

${ }^{24}$ Garaigordobil, Maite, Carmen Maganto (2011), rEmpatía y resolución de conflictos durante la infancia y la adolescenciaò Revista Latinoamericana de Psicología, vol. 43, núm. 2, Colombia, pp. 255-266.

${ }^{25}$ Choi, Jiyoung, Johnson, W. David, Johnson Roger (2011), r̃Relationships among

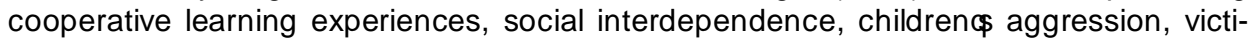
mization, and prosocial behavioursò Journal of Applied Social Psychology, v. 41, n. 4. US. doi: 10.1111/j.1559-1816.2011.00744.x.

${ }^{26}$ Zych, Izabela, María Beltrán Catalán, Rosario Ortega Ruiz, Vicente J. Llorent (2018), ñSocial and emotional competencies in adolescents involved in different bullying an cyberbullying rolesò Revista de Psicodidáctica, vol. 23, núm. 2, España, pp. 86-93.

${ }_{27}$ Zischka, Lorna (2018), ñThe link between prosocial (giving) behaviours and social cohesionò Revista Internacional de Sociología, vol. 76, núm. 1, Córdoba, España. e082. https://doi.org/10.3989/ris.2018.76.1.15.125

${ }^{28}$ Van Ryzin, Mark J., Cary J. Roseth (2018), r̃Cooperative learning in middle school: A means to improve peer relations and reduce victimization, bullying, and related outcomesò Journal of Educational Psychology, NY, IN PRESS. Doi: 10.1037/edu0000265.

${ }^{29}$ León, Benito et al. (2016), op. cit. 
Además, tal como dice Galindo, ${ }^{30}$ toda interacción humana requiere de un esfuerzo. r̃ Cuando estamos en presencia de otro(s) actor(es) incluso la actividad más banal adquiere rango de aventura. Y es que, en el ámbito de la interacción, todas las actividades requieren de nuestro esfuerzo para poder ser llevadas a cabo con éxitoò Consideramos necesario educar a nuestros estudiantes en esta tarea del esfuerzo para que las relaciones entre ellos sean correctas, que hagan un esfuerzo por escuchar, entender y respetar las ideas de los demás, y reflexionar sobre ellas, ya que en más de una ocasión se darán cuenta de que son mejores que la suya propia. लें Esto derivará en un aprendizaje muy significativo.

Además, el bienestar personal no existe sin una actitud de compromiso y responsabilidad por el bienestar del grupo. Somos seres sociales; el mundo de cada uno de nosotros se configura a través de las relaciones interpersonales y del hacer correcto y constante de cada uno. No somos seres estáticos y nos movemos en un entorno de responsabilidad ética, respetando a los demás y así creamos espacios donde hacemos cosas juntos y el resultado de ese entorno dependerá de la r̃co-inspiración participativa en un proyecto comúnò ${ }^{31}$

Los grupos de trabajo colaborativo pueden establecer en las personas los sentimientos de pertenencia, aceptación y cuidado, además de aportar recursos a través de los cuales poder solventar las situaciones problemáticas de la vida. La vinculación al grupo es esencial para adaptarse saludablemente a la vida social..$^{32}$

Con todo ello, consideramos que el aprendizaje colaborativo puede reducir el nivel de agresividad en las personas y ayudar en el plan de esfuerzo que han de adoptar para conseguir una fructífera formación, bienestar personal y social, y éxito en la tarea. Si esta formación se adquiere en la etapa escolar, estaremos consiguiendo que, en la vida adulta, su vida profesional sea exitosa.

${ }^{30}$ Galindo, Jorge (2015), ĩErving Goffman y el orden de la interacciónò Acta Sociológica, núm. 66, CES, FCPyS UNAM, p. 17.

${ }^{31}$ Humberto Maturana (1996) en Trujillo Vargas, John (1998), ñTrabajo en equipo, una propuesta para los procesos de enseñanza ï aprendizajeò IV Congreso RIBIE, Brasilia.

32 Johnson, David, Roger Johnson, Edythe Holubec (1999), El aprendizaje cooperativo en el aula, Paidós, Buenos Aires. 
En el cruce entre el trabajo en equipo y el tipo de centro, no se constataron diferencias estadísticamente significativas, aunque fueron los alumnos del centro público los que reflejaron valores más elevados en trabajo grupal. Lledó y Arnaiz ${ }^{33}$ señalan que los docentes de centros educativos públicos suelen optar, en mayor medida, por actividades de formación continua usando estrategias metodológicas variadas, lo que puede explicar que en los centros públicos se trabaje más la modalidad del trabajo en equipo. ${ }^{34}$

Con respecto a la relación entre trabajo en grupo y curso, se manifiestan diferencias con una puntuación media superior en 6 을 de primaria respecto a $1^{\circ}$ de ESo. Se revela que entre más jóvenes sean los alumnos, es mayor su motivación por el trabajo en grupo. A esta misma conclusión llegaron Kyndt, Raes, Lismont, Timmers, Cascallar y Dochy ${ }^{35}$ quienes aseguraron que es mayor el interés por esta estrategia en niveles educativos inferiores. Tal vez es por el incremento considerable del volumen de contenidos en educación secundaria y por la falta de tiempo para su impartición, pero queda claro que es en educación primaria cuando más frecuentemente se hace uso de la metodología grupal de trabajo. En esta línea, Garaigodobil y Maganto ${ }^{36}$ revelan en sus investigaciones que las estrategias cooperativas positivas no mejoran con la edad. Pero este hecho nos abre preguntas: ¿es que los alumnos de secundaria pierden interés por esta estrategia metodológica porque no es muy utilizada en dicha etapa? o tal vez, el enfoque que el profesor da a su asignatura en secundaria, ¿no conduce a la utilización del trabajo en equipo como estrategia? ¿Puede ser que los alumnos hayan cambiado sus objetivos al acceder a secundaria, miren

${ }^{33}$ Lledó Carreres, Asunción, Pilar Arnaiz Sánchez (2016), ĩEvaluación de las prácticas educativas del profesorado de los centros escolares: indicadores de mejora desde la educación inclusivaò REICE, vol. 8, núm. 5, Madrid, pp. 97-109.

${ }^{34}$ Alfonso-Benlliure, Vicente, Martha Valadez (2013), ñntereses académicos, extraescolares y desempeño creativo en educación primaria según género, tipo de escuela y contexto escolarò Revista Mexicana de Psicología, vol. 30, núm. 1, Ciudad de México, pp. 41-52.

${ }^{35}$ Kyndt, Eva, Elisabeth Raes, Bart Lismont, Fran Timmers, Eduardo Cascallar, Filip Dochy (2013), ñA meta-analysis of the effects of face-to-face cooperative learning. Do recent studies falsify or verify earlier findings?ò Educational Research Review, n. 10, Germany, pp. 133-149.

${ }^{36}$ Garaigordobil, Maite, Carmen Maganto (2011), rÉmpatía y resolución de conflictos durante la infancia y la adolescenciaò Revista Latinoamericana de Psicología, vol. 43, núm. 2, Colombia, pp. 255-266. 
sus estudios con perspectivas profesionales, comiencen a labrarse su futuro individualmente, y por esta razón la estrategia del trabajo en equipo no tenga cabida o la tenga menos? La respuesta a todas estas preguntas la podremos dar después de realizar otra investigación que lo resuelva.

De momento, en este trabajo podemos decir que a medida que los alumnos crecen en edad, disminuye el interés y la práctica de la estrategia metodológica del trabajo en equipo. De esta manera y con el avance de los años, ¿podrán estos alumnos estar preparados para llevar a cabo un trabajo en equipo en su vida profesional? ¿Habrán adquirido en primaria las habilidades sociales necesarias y habrán sido capaces de mantenerlas a lo largo de su vida? Estas preguntas necesitan también de otra investigación para ser respondidas. De todos modos, consideramos que, debido a la importancia del trabajo en equipo, no se debería perder ni disminuir su práctica en secundaria ni en ninguna otra etapa educativa.

Con todo lo dicho, consideramos que es necesario destacar las limitaciones de este trabajo de investigación. El diseño del estudio es de tipo descriptivo lo que no permite establecer relaciones de causa-efecto. Con este estudio hemos conocido el nivel de aprendizaje y práctica que tienen los alumnos de $6^{\circ}$ de primaria y $1^{\circ}$ de ESo en la estrategia metodológica del trabajo colaborativo. Sin embargo, han surgido muchas preguntas que requieren de otro tipo investigación donde la relación causa-efecto sea viable.

El tamaño de la muestra es representativo para los alumnos de 6ํ de primaria y $1^{\circ}$ de ESO, pero no incluye otros cursos, lo que aportaría una gran riqueza a la investigación.

Además, ante la importancia del tema, hubiese sido de interés utilizar otros instrumentos que hubiesen permitido conocer con más detalle y profundidad, los niveles de adquisición del aprendizaje colaborativo.

Todos estos aspectos están siendo considerados como perspectiva de investigaciones futuras.

Para finalizar, se pueden establecer las siguientes conclusiones en este trabajo de investigación:

- Las chicas están más motivadas, son más responsables y se implican más en los trabajos en grupo que los chicos.

- A mayor edad, curso superior, disminuye la motivación y la implicación por el trabajo en grupo. 
- Los alumnos de los centros públicos se implican algo más en las tareas grupales que los de los centros privados, aunque las diferencias no son significativas.

Por tanto, viendo los resultados obtenidos, se puede sugerir que hay que reforzar desde edades tempranas el trabajo en grupo y continuar utilizándola en todas las etapas educativas. Asimismo, los varones necesitan una mayor intervención para que se impliquen de forma adecuada y responsable en el trabajo colaborativo.

A pesar de las limitaciones del trabajo colaborativo, las ventajas que presenta son abrumadoras e imprescindibles para la profesionalización y vida social de las personas en el siglo xxI. Por tanto, debemos apostar por mejorar la formación del profesorado para que puedan solventar los inconvenientes de esta metodología y conseguir para el alumnado todas las ventajas que una enseñanza-aprendizaje colaborativo aporta.

\section{Bibliografía}

Alfonso-Benlliure, Vicente, Martha Valadez (2013), ñntereses académicos, extraescolares y desempeño creativo en educación primaria según género, tipo de escuela y contexto escolarò Revista Mexicana de Psicología, vol. 30, núm. 1, Ciudad de México.

Andreu-Andrés, María Ángeles, Miguel García-Casas, (2008), Ĩa autoevaluación del trabajo en grupo en la clase de lengua extranjera: investigación de campoò La evaluación en el aprendizaje y la enseñanza del español como LE/L2, Susa Pastor y Santiago Roca Editores, Universidad de Alicante, Alicante, España.

Barbería, Garbiñe (2012), ñTrabajo Colaborativoò Trabajo Fin de Master en la Universidad Pública de Navarra, Master Universitario en Formación del Profesorado en Educación Secundaria, en: https://academica e.unavarra.es/bitstream/handle/2454/15005/58502_Barberia $\% 20 \mathrm{P} \%$ C3\%A9rez\%2C\%20Garbi\%C3\%B1e.pdf? sequence=1 \&isAllowed $=\mathrm{y}$

Bernal Vázquez, Julia, Amaya Epelde Larrañaga, Miguel Ángel Gallardo Vigil, África Rodríguez Blanco (2012), ña enseñanza colaborativa en 
Educación Primaria. Un estudio sobre el trabajo conjunto de los docentes de música e inglés para mejorar la formación del alumnadoò DEDICA, Revista de Educação e Humanidades, núm. 2, Portugal.

Chacón-Cuberos, Ramón, Manuel Castro-Sánchez, Gloria González-Campos, Félix Zurita-Ortega (2018), ñictimización en la escuela, ocio digital e irritabilidad: análisis mediante ecuaciones estructuralesò RELIEVE, vol. 24, núm.1, Valencia. Doi: 10.7203/relieve.24.1.12614.

Choi, Jiyoung, David W. Johnson, Roger Johnson (2011), ז̃Relationships among cooperative learning experiences, social interdependence,

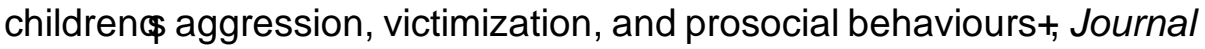
of Applied Social Psychology, v. 41, n. 4. US. Doi: 10.1111/j.1559-1816. 2011.00744.x.

Dávila Martín, Estefanía (2016), ĩRápido a ninguna parte. Consideraciones en torno a la aceleración del tiempo socialò Acta Sociológica, núm. 69, CES, FCPYS, UNAM.

Froiland, John Mark, Emily Oros (2014), ñntrinsic motivation, perceived competence and classroom engagement as longitudinal predictors of adolescent reading achievementò Educational Psychology, v. 34, n. 2, NY.

Galindo, Jorge (2015), r̃Erving Goffman y el orden de la interacciónò Acta Sociológica, núm. 66, CES, FCPys UnAM.

Garaigordobil, Maite, Carmen Maganto (2011), íEmpatía y resolución de conflictos durante la infancia y la adolescencià̀ Revista Latinoamericana de Psicología, vol. 43, núm. 2, Colombia.

García-Valcárcel, Ana, Verónica Basilotta, Camino López (2014), ñLas TIC en el aprendizaje colaborativo en el aula de Primaria y Secundariaò Comunicar, vol. 42, núm. 21, España.

Goikoetxea, Edurne, Gema Pascual (2002), r̃Aprendizaje cooperativo: Bases teóricas y hallazgos empíricos que explican su eficaciaò Educación XXI, núm. 5.

Gómez Múgica, Aleida y Acosta Rodríguez, Heriberto (2003). ñAcerca del trabajo en grupos o equiposò ACIMED, vol. 11, núm.6, Cuba.

Johnson, David, Roger Johnson, Edythe Holubec (1999), El aprendizaje cooperativo en el aula, Paidós, Buenos Aires.

Kibirige, Israel, Jeridah Lehong Moyahabo (2016), ñThe effect of cooperative learning on grade 12 learnersôperformance in projectile motions, 
South Africaò Eurasia Journal of Mathematics, Science \& Technology Education, v. 12, n. 9, uk.

Kyndt, Eva, Elisabeth Raes, Bart Lismont, Fran Timmers, Eduardo Cascallar, Filip Dochy (2013), r̃A meta-analysis of the effects of face-toface cooperative learning. Do recent studies falsify or verify earlier findings?ò Educational Research Review, n. 10, Germany,

León, Benito, Margarita Gozalo, María Isabel Polo (2012), ñAprendizaje cooperativo y acoso entre igualesò Infancia y aprendizaje, vol. 35, núm. 1, España.

León, Benito, María Isabel Polo, Margarita Gozalo, Santiago Mendo (2016), ז̃Relevancia del aprendizaje cooperativo sobre los diferentes perfiles de la dinámica bullying: un análisis mediante pruebas de tamaño del efectoò Anales de Psicología, vol. 32, núm. 1, Murcia, España. doi: 10.6018/ analesps.32.1.183141.

Lledó Carreres, Asunción, Pilar Arnaiz Sánchez (2016), ŕEvaluación de las prácticas educativas del profesorado de los centros escolares: indicadores de mejora desde la educación inclusivaò REICE, Revista Iberoamericana sobre Calidad, Eficacia y Cambio en Educación, vol. 8, núm. 5, Madrid.

Merino-Marban, Rafael, Daniel Mayorga-Vega, Emilio Fernández-Rodríguez, Francisco Vera Estrada, Jesús Viciana (2015), íEffect of a physical education-based stretching programme on sit-andreach score and its posterior reduction in elementary schoolchildrenò European Physical Education Review, núm. 21, Uk.

Moreno Peláez, Florentina (1997), r̃aprendizaje organizativo y generación de competenciasò Revista Española de Investigaciones Sociológicas, núm. 77 , cIs, Madrid.

Pang, Christopher, Jesslyn Lau, Chong Poh Seah, Linda Cheong, Audrey Low (2018), î́socially Challenged Collaborative Learning of Secondary School Students in Singaporeò Education Sciences, v. 8, n. 24, Switzerland.

Piaget, Jean (1969), Psicología y Pedagogía, Ariel, Barcelona. PISA (2015), en: http://www.mecd.gob.es/dctm/inee/internacional/pisa2015/ pisa2015preliminarok.pdf? documentld $=0901$ e72b8228b93c

Polo, María Isabel, Santiago Mendo, Fernando Fajardo, Benito León (2017), ñna intervención en aprendizaje cooperativo sobre el perfil del 
observador en la dinámica bullyingò Universitas Psychologica, vol. 16, núm. 1, Colombia.

Rosenfield, Sylvia, Markeda Newell, Scott Zwolski, Lauren E. Benishek (2018), rEvaluating problem-solving teams in Kï 12 Schools: Do they work? à American Psychological Association, v. 73, n. 4. Washington, D.C

Trujillo Vargas, John (1998), ñTrabajo en equipo, una propuesta para los procesos de enseñanza ï aprendizajeò ıv Congreso RIBIE, Brasilia, 1998. Van Ryzin, Mark J., Cary J. Roseth (2018), r̃Cooperative learning in middle school: A means to improve peer relations and reduce victimization, bullying, and related outcomesò Journal of Educational Psychology, IN PRESS. doi: $10.1037 /$ edu0000265.

Zischka, Lorna (2018), ñThe link between prosocial (giving) behaviours and social cohesionò Revista Internacional de Sociología, vol. 76, núm. 1, Córdoba, España. e082. doi: https://doi.org/10.3989/ris.2018.76.1.15.125 Zych, Izabela, María Beltrán Catalán, Rosario Ortega Ruiz, Vicente J. Llorent (2018), r̃ Social and emotional competencies in adolescents involved in different bullying an cyberbullying rolesò Revista de Psicodidáctica, v. 23, n. 2, España. 
El tRabajo COLAbORATIVO EN PRIMARIA Y SECUNDARIA: DIFERENCIAS SEGÚN...

\section{Anexo 1}

\section{Tabla I}

Trabajo en grupo según el sexo

\begin{tabular}{|c|c|c|c|c|c|c|}
\hline Items TG.V & Sexo & Nunca & $\begin{array}{c}\text { Algunas } \\
\text { veces }\end{array}$ & $\begin{array}{c}\text { Bastantes } \\
\text { veces }\end{array}$ & Siempre & $\mathrm{P}$ \\
\hline \multirow{3}{*}{ TG.V.1 } & Hombre & $7.8 \% \quad(n=8)$ & $19.4 \% \quad(n=20)$ & $26.2 \% \quad(n=27)$ & $45.6 \%(n=47)$ & \multirow{3}{*}{0.294} \\
\hline & Mujer & $2.4 \%(n=3)$ & $18.5 \%(n=23)$ & $29.0 \% \quad(n=36)$ & $50.0 \%(n=62)$ & \\
\hline & Total & $4.8 \%(n=11)$ & $18.9 \%(n=43)$ & $27.8 \%(n=63)$ & $48.0 \%(n=109)$ & \\
\hline \multirow{3}{*}{ TG.V.2 } & Hombre & $8.7 \%(n=8)$ & $11.7 \% \quad(n=12)$ & $27.2 \%(n=28)$ & $52.4 \% \quad(n=54)$ & \multirow{3}{*}{$0.016^{*}$} \\
\hline & Mujer & $0.0 \% \quad(n=0)$ & $14.5 \%(n=18)$ & $24.2 \%(n=30)$ & $61.3 \%(n=76)$ & \\
\hline & Total & $3.5 \%(n=8)$ & $13.2 \%(n=30)$ & $25.6 \% \quad(n=58)$ & $57.3 \%(n=130)$ & \\
\hline \multirow{3}{*}{ TG.V.3 } & Hombre & $6.8 \%(n=7)$ & $17.5 \%(n=18)$ & $27.2 \%(n=28)$ & $47.6 \%(n=49)$ & \multirow{3}{*}{$0.033^{*}$} \\
\hline & Mujer & $0.8 \%(n=1)$ & $11.3 \%(n=14)$ & $37.9 \%(n=47)$ & $50.0 \%(n=62)$ & \\
\hline & Total & $3.5 \%(n=8)$ & $14.1 \%(n=32)$ & $33.0 \%(n=75)$ & $48.9 \%(n=111)$ & \\
\hline \multirow{3}{*}{ TG.V.4 } & Hombre & $2.9 \%(n=3)$ & $11.8 \%(n=12)$ & $28.4 \%(n=29)$ & $55.9 \%(n=57)$ & \multirow{3}{*}{0.300} \\
\hline & Mujer & $1.6 \%(n=2)$ & $8.1 \%(n=10)$ & $21.8 \%(n=27)$ & $68.5 \%(n=85)$ & \\
\hline & Total & $2.2 \%(n=5)$ & $9.7 \%(n=22)$ & $24.8 \%(n=56)$ & $62.8 \%(n=142)$ & \\
\hline \multirow{3}{*}{ TG.V.5 } & Hombre & $3.9 \%(n=4)$ & $27.2 \%(n=28)$ & $39.8 \% \quad(n=41)$ & $28.2 \%(n=29)$ & \multirow{3}{*}{0.668} \\
\hline & Mujer & $3.2 \%(n=4)$ & $23.4 \%(n=29)$ & $38.7 \% \quad(n=48)$ & $34.7 \%(n=43)$ & \\
\hline & Total & $3.5 \%(n=8)$ & $25.1 \%(n=57)$ & $39.2 \%(n=89)$ & $31.7 \%(n=72)$ & \\
\hline \multirow{3}{*}{ TG.V.6 } & Hombre & $15.5 \%(n=16)$ & $28.2 \%(n=29)$ & $30.1 \% \quad(n=31)$ & $25.2 \%(n=26)$ & \multirow{3}{*}{$0.055^{\star}$} \\
\hline & Mujer & $6.5 \%(n=8)$ & $26.6 \%(n=33)$ & $27.4 \% \quad(n=34)$ & $39.5 \%(n=49)$ & \\
\hline & Total & $10.6 \%(n=24)$ & $27.3 \%(n=62)$ & $28.6 \%(n=65)$ & $33.0 \%(n=75)$ & \\
\hline \multirow{3}{*}{ TG.V.7 } & Hombre & $30.1 \% \quad(n=31)$ & $27.2 \%(n=28)$ & $17.5 \%(n=18)$ & $24.3 \%(n=25)$ & \multirow{3}{*}{0.338} \\
\hline & Mujer & $21.0 \%(n=26)$ & $36.3 \%(n=45)$ & $17.7 \% \quad(n=22)$ & $25.0 \%(n=31)$ & \\
\hline & Total & $25.1 \% \quad(n=57)$ & $32.2 \%(n=73)$ & $17.6 \% \quad(n=40)$ & $24.7 \%(n=56)$ & \\
\hline \multirow{3}{*}{ TG.V.8 } & Hombre & $7.8 \%(n=8)$ & $12.6 \%(n=13)$ & $43.7 \% \quad(n=45)$ & $35.0 \%(n=36)$ & \multirow{3}{*}{$0.020^{*}$} \\
\hline & Mujer & $3.2 \%(n=4)$ & $21.0 \%(n=26)$ & $28.2 \%(n=35)$ & $47.6 \%(n=59)$ & \\
\hline & Total & $5.3 \%(n=12)$ & $17.2 \%(n=39)$ & $35.2 \%(n=80)$ & $41.9 \%(n=95)$ & \\
\hline \multirow{3}{*}{ TG.V.9 } & Hombre & $10.7 \%(n=11)$ & $30.1 \%(n=31)$ & $31.1 \% \quad(n=32)$ & $27.2 \% \quad(n=28)$ & \multirow{3}{*}{$0.035^{*}$} \\
\hline & Mujer & $3.2 \%(n=4)$ & $21.0 \%(n=26)$ & $39.5 \%(n=49)$ & $36.3 \%(n=45)$ & \\
\hline & Total & $6.6 \%(n=15)$ & $25.1 \%(n=57)$ & $35.7 \% \quad(n=81)$ & $32.2 \%(n=73)$ & \\
\hline \multirow{3}{*}{ TG.V.10 } & Hombre & $7.8 \%(\mathrm{n}=8)$ & $19.4 \%(n=20)$ & $31.1 \% \quad(n=32)$ & $40.8 \%(n=42)$ & \multirow{3}{*}{0.070} \\
\hline & Mujer & $2.4 \%(n=3)$ & $10.5 \%(n=13)$ & $33.9 \%(n=42)$ & $52.4 \%(n=65)$ & \\
\hline & Total & $4.8 \%(n=11)$ & $14.5 \%(n=33)$ & $32.6 \%(n=74)$ & $47.1 \%(n=107)$ & \\
\hline \multirow{3}{*}{ TG.V.11 } & Hombre & $6.8 \%(n=7)$ & $32.0 \%(n=33)$ & $28.2 \%(n=29)$ & $32.0 \%(n=33)$ & \multirow{3}{*}{0.681} \\
\hline & Mujer & $5.6 \%(n=7)$ & $37.1 \%(n=46)$ & $23.4 \% \quad(n=29)$ & $33.9 \%(n=42)$ & \\
\hline & Total & $6.2 \%(n=14)$ & $34.8 \%(n=79)$ & $25.6 \%(n=58)$ & $33.0 \%(n=75)$ & \\
\hline \multirow{3}{*}{ TG.V.12 } & Hombre & $5.8 \%(n=6)$ & $20.4 \%(n=21)$ & $27.2 \% \quad(n=28)$ & $45.6 \%(n=47)$ & \multirow{3}{*}{0.280} \\
\hline & Mujer & $1.6 \%(n=2)$ & $17.7 \%(n=22)$ & $33.9 \% \quad(n=42)$ & $46.8 \%(n=58)$ & \\
\hline & Total & $3.5 \%(n=8)$ & $18.9 \%(n=43)$ & $30.8 \%(n=70)$ & $46.3 \%(n=105)$ & \\
\hline
\end{tabular}


Amaya Epelde L. / Ramón Chacón C. / José Antonio Oñederra R.

\begin{tabular}{|c|c|c|c|c|c|c|}
\hline \multirow{3}{*}{ TG.V.13 } & Hombre & $8.7 \%(n=9)$ & $11.7 \%(n=12)$ & $20.4 \% \quad(n=21)$ & $57.3 \% \quad(n=59)$ & \\
\hline & Mujer & $3.2 \%(n=4)$ & $16.9 \%(n=21)$ & $16.1 \%(n=20)$ & $63.7 \%(n=79)$ & \\
\hline & Total & $5.7 \%(n=13)$ & $14.5 \%(n=33)$ & $18.1 \%(n=41)$ & $60.8 \%(n=138)$ & \multirow{4}{*}{0.268} \\
\hline \multirow{3}{*}{ TG.V.14 } & Hombre & $9.7 \% \quad(n=10)$ & $19.4 \% \quad(n=20)$ & $19.4 \% \quad(n=20)$ & $49.5 \%(n=51)$ & \\
\hline & Mujer & $6.5 \%(n=8)$ & $13.7 \% \quad(n=17)$ & $21.8 \% \quad(n=27)$ & $58.1 \%(n=72)$ & \\
\hline & Total & $7.9 \% \quad(n=18)$ & $16.3 \%(n=37)$ & $20.7 \% \quad(n=47)$ & $54.2 \%(123)$ & \\
\hline
\end{tabular}

TG.V.1: contribuyes a que la comunicación entre los miembros del grupo sea clara y fluida; TG.V.2: tienes claras tus responsabilidades como miembro del equipo; TG.V.3: contribuyes a que exista un clima de trabajo agradable; TG.V.4: escuchas las opiniones de los demás al trabajar en grupo; TG.V.5: escuchan los demás tus opiniones al trabajar en grupo; TG.V.6: gestionas los conflictos de manera abierta; TG.V.7: has gestionado los conflictos pensando más en las ideas que en las personas; TG.V.8: ayudas a generar alternativas de solución ante los problemas; TG.V.9: analizas en grupo las posibles consecuencias de cada alternativa; TG.V.10: participas en el desarrollo en grupo de planes alternativos de trabajo; TG.V.11: aceptas las críticas de forma positiva; TG.V.12: aceptas las decisiones que se toman en el grupo; TG.V.13: te gusta e ilusiona trabajar en grupo; TG.V.14: creo que aprendo más trabajando en grupo que trabajando individualmente.

Anexo 2

Tabla II

Relación entre trabajo en grupo y curso

\begin{tabular}{|c|c|c|c|c|c|c|}
\hline $\begin{array}{l}\text { Ítems } \\
\text { TG.V }\end{array}$ & Curso & Nunca & $\begin{array}{c}\text { Algunas } \\
\text { veces }\end{array}$ & $\begin{array}{c}\text { Bastantes } \\
\text { veces }\end{array}$ & Siempre & $P$ \\
\hline \multirow{3}{*}{ TG.V.4 } & $\begin{array}{l}\text { Primero } \\
\text { de ESO }\end{array}$ & $1.2 \%(n=2)$ & $10.8 \%(n=18)$ & $28.3 \% \quad(n=47)$ & $59.0 \%(\mathrm{n}=98)$ & \multirow{3}{*}{0.072} \\
\hline & $\begin{array}{l}\text { Sexto de } \\
\text { Primaria }\end{array}$ & $5.0 \%(n=3)$ & $6.7 \%(n=4)$ & $15.0 \% \quad(n=9)$ & $73.3 \% \quad(n=44)$ & \\
\hline & Total & $2.2 \%(n=5)$ & $9.7 \%(n=22)$ & $24.8 \%(n=56)$ & $62.8 \%(n=142)$ & \\
\hline \multirow{3}{*}{ TG.V.7 } & $\begin{array}{l}\text { Primero } \\
\text { de ESO }\end{array}$ & $26.9 \% \quad(n=45)$ & $35.3 \% \quad(n=59)$ & $18.6 \%(n=31)$ & $18.6 \% \quad(n=31)$ & \multirow{3}{*}{$0.011^{*}$} \\
\hline & $\begin{array}{l}\text { Sexto de } \\
\text { Primaria }\end{array}$ & $20.0 \%(n=12)$ & $23.3 \% \quad(n=14)$ & $15.0 \% \quad(n=9)$ & $41.7 \% \quad(n=25)$ & \\
\hline & Total & $25.1 \%(n=57)$ & $32.2 \%(n=73)$ & $17.6 \%(n=40)$ & $24.7 \%(n=56)$ & \\
\hline \multirow{3}{*}{ TG.V.10 } & $\begin{array}{l}\text { Primero } \\
\text { de ESO }\end{array}$ & $48 \%(n=8)$ & $18.6 \% \quad(n=31)$ & $32.3 \%(n=54)$ & $43.1 \% \quad(n=72)$ & \multirow{3}{*}{0.077} \\
\hline & $\begin{array}{l}\text { Sexto de } \\
\text { Primaria }\end{array}$ & $5.0 \%(n=3)$ & $3.3 \%(n=2)$ & $33.3 \%(n=20)$ & $58.3 \% \quad(n=35)$ & \\
\hline & Total & $4.8 \%(n=11)$ & $14.5 \%(n=33)$ & $32.6 \%(n=74)$ & $47.1 \%(n=107)$ & \\
\hline
\end{tabular}

TG.V.4: escuchas las opiniones de los demás al trabajar en grupo; TG.V.7: has gestionado los conflictos pensando más en las ideas que en las personas; TG.V.10: participas en el desarrollo en grupo de planes alternativos de trabajo. 